\title{
Effet du Manganèse sur la Croissance de la Patate Douce (Ipomoea batatas L.) au Gabon
}

\author{
Alexis Nicaise Lepengue, \\ Laboratoire de Physiologie Végétale et Protection des Plantes, Unité de \\ Recherche Agrobiologie, Université des Sciences et Techniques de Masuku, \\ Franceville-Gabon \\ Ephrem Nzengue,
}

Institut de Recherche en Écologie Tropicale du Centre National de Recherches Scientifiques et Technologiques, Libreville-Gabon, Laboratoire de Physiologie Végétale et Protection des Plantes, Unité de Recherche Agrobiologie Université des Sciences et Techniques de Masuku, Franceville-Gabon

Stéphane Mombo, Christophe Yene Essougou, Dhert Souviens Tshi-Tshi Ontod, Aurélien Mokea-Niaty, Bertrand Mbatchi,

Laboratoire de Physiologie Végétale et Protection des Plantes, Unité de Recherche Agrobiologie, Université des Sciences et Techniques de Masuku, Franceville-Gabon

Doi:10.19044/esj.2019.v15n24p281 URL:http://dx.doi.org/10.19044/esj.2019.v15n24p281

\section{Résumé}

La contamination des sols de la ville minière de Moanda, au Sud-Est du Gabon est caractérisée par de fortes concentrations de manganèse. De ce fait, ce travail vise à analyser l'impact de diverses concentrations de manganèse dans les sols sur la croissance et le développement de la patate douce. Les semences de la plante ont été obtenues sur le marché local. Les tiges ont été cultivées en serre sur 5 milieux dont 4 enrichis en manganèse et 1 non enrichi. L'effet manganifère a été évalué par la mesure des paramètres biochimiques et physico-chimiques (chlorophylle totale, matière sèche, sucres totaux). Les effets des 5 traitements ont été comparés sur le logiciel Statistica 7.2. à partir d'une Anova. Les résultats montrent que les fortes concentrations $(25 \%, 50 \%, 75 \%$ et $100 \%)$ affectent les caractéristiques morphométriques de la patate douce. Les concentrations à $25 \%$ de Mn entraînent une augmentation des paramètres et une diminution de ces derniers à $50 \%$ et $75 \%$ de $\mathrm{Mn}$, par 
rapport au témoin. Les concentrations à 100\% de Mn inhibent totalement la croissance et le développement de la patate douce.

Mots clés : Manganèse, Ipomoea batatas, Moanda, Chlorophylle totale, Matière sèche, Sucres totaux

\title{
Effect of Manganese on Sweet Potato (Ipomoea batatas L.) Growth in Gabon
}

\section{Alexis Nicaise Lepengue,}

Laboratoire de Physiologie Végétale et Protection des Plantes, Unité de Recherche Agrobiologie, Université des Sciences et Techniques de Masuku,

Franceville-Gabon

Ephrem Nzengue,

Institut de Recherche en Écologie Tropicale du Centre National de Recherches Scientifiques et Technologiques, Libreville-Gabon, Laboratoire de Physiologie Végétale et Protection des Plantes, Unité de Recherche Agrobiologie

Université des Sciences et Techniques de Masuku, Franceville-Gabon

Stéphane Mombo, Christophe Yene Essougou, Dhert Souviens Tshi-Tshi Ontod, Aurélien Mokea-Niaty, Bertrand Mbatchi,

Laboratoire de Physiologie Végétale et Protection des Plantes, Unité de Recherche Agrobiologie, Université des Sciences et Techniques de Masuku, Franceville-Gabon

\begin{abstract}
Soil contamination in the mining town of Moanda, in southwestern of Gabon, is characterized by high concentrations of Manganese. This work aims to analyze the impact of various manganese concentrations in soils on sweet potato growth and development. The plant's seeds were obtained on the local market. The stems were grown in greenhouses in 5 media, 4 of which were enriched with manganese and 1 not enriched. The manganiferous effect was assessed by measuring biochemical and physico-chemical parameters (total chlorophyll, dry matter, total sugars). The effects of the 5 treatments were compared on the Statistica 7.2. software from an Anova. The results show that
\end{abstract}


high concentrations $(25 \%, 50 \%, 75 \%$ and $100 \%)$ affect the morphometric characteristics of the sweet potato. Concentrations at $25 \% \mathrm{Mn}$ increase the parameters and decrease them to $50 \%$ and $75 \% \mathrm{Mn}$, compared to the control. The $100 \%$ Mn concentrations totally inhibit the growth and development of the sweet potato.

Keywords: Manganese, Ipomoea batatas, Moanda, Total chlorophyll, Dry matter, Total sugars

\section{Introduction}

Le manganèse devient progressivement la principale source de devise issue des activités minières au Gabon, devant l'or, le diamant et après le pétrole (Ndala et Moussone, 2011 ; Aterianus-owanga et Debain, 2016 ; COMILOG, 2018). Exploité depuis 1957 par la Compagnie Minière de l'Ogooué (COMILOG), le Gabon est le deuxième producteur mondial de manganèse avec 25\% de la demande mondiale (Ndala et Moussone, 2011 ; Clarke et Upson, 2017 ; COMILOG, 2018). D’abord localisés à Moanda au Sud-Est du Gabon, les gisements manganifères ont également été découverts et mis en exploitation depuis peu à Franceville, à Okondja (Sud-Est) et à Maboumi (Centre-Ouest du pays). A Moanda, l'exploitation est assurée par des multinationales, dont le principal investisseur est le groupe Français Eramet (Ontod et al., 2013 ; Comilog, 2018). Le besoin de diversification de l'économie du pays a favorisé l'installation d'autres investisseurs étrangers notamment, les groupes indiens Mohan Exports India PVT et Manganese Ore India Ltd (MOIL) et la Chine à travers la Compagnie Industrielle et Commerciale des Mines de Huazhoou (CICMHZ) qui s'intéressent à l'exploitation d'une partie des gisements gabonais (Ndala et Moussone, 2011). Du point de vue géologique, les gisements de manganèse sont enfouis à une profondeur moyenne de 7 mètres. L'excavation des sols de couverture et l'exploitation à ciel ouvert de ce minerai provoque la dispersion des roches et leurs poussières, occasionnant la pollution de l'air (Xiong et al., 2014 ; Mombo et al., 2015) et du sol (Boupassia, 2004). Ces pollutions sont susceptibles d'affecter tous les compartiments de l'écosystème notamment les cours d'eau, la faune et les végétaux (Lebas, 2010 ; Ontod et al., 2013 ; Nguéma et al., 2014 ; Goix et al., 2015 ; Leveque et al., 2015 ; Ontod et al., 2015 ; Mombo et al., 2018 ; Messi-Me, 2018 ; Mokea-Niaty, 2019). En effet, les travaux de recherche réalisés dans la zone ont révélé que les poussières de manganèse perturbaient, par leur toxicité, la croissance et le développement des plantes de Hibiscus sabdariffa (Ontod et al., 2013 ; Mavoungou et al., 2015). Ces résultats ont été corroborés par ceux de Ontod (2017), Mombo et al. (2017) et Nzengue et al. (2019) qui ont montré que les fortes teneurs de manganèse des sols de Moanda pouvaient inhiber la croissance des tiges et des 
racines du manioc (Manihot esculenta Crantz) et de l'arachide (Arachis hypogea L.). Elles avaient également des effets antagonistes sur la production de biomasse et l'activité photosynthétique de Alchornea cordifolia (MokeaNiaty, 2019).

Par ailleurs, au Gabon, les plantes à tubercules constituent les principales sources alimentaires du pays. Il s'agit notamment du manioc, de la patate douce et du taro. Ces plantes sont abondamment cultivées dans les plantations rurales proches des zones d'exploitation du manganèse telle que la ceinture agricole du plateau Bangombé à Moanda (Eba et al., 2007 ; Mombo et al., 2017). Si les impacts des poussières manganifères sur les plantes de manioc ont déjà été étudiés, les réactions d'autres plantes à tubercules, notamment la patate douce (Ipomoea batatas, Convolvulaceae), vis-à-vis de ce minerai restent inconnues à nos jours. Cette étude vise à mesurer l'évolution de quelques paramètres morphométriques et biochimiques de la patate douce, cultivée sur des substrats suivant un gradient de concentrations en manganèse afin de fournir des informations sur l'impact de l'exploitation minière sur les plantes à tubercules de la région de Moanda au Sud-Est du Gabon.

\section{Matériel et méthodes Matériel}

Le matériel végétal utilisé dans cette étude était constitué des plants de patate douce (Ipomoea batatas; Convolvulaceae). Les plants, achetés au marché municipal de Potos, correspondaient à la variété locale "Elengi' à tubercules jaunes, avec des entre-nœuds très courts et des feuilles simples à pétiole vert et nervures pourpres. Le manganèse utilisé a été offert par la Compagnie Minière de l'Ogooué (COMILOG) installée à Moanda. Les sols témoins ont été prélevés sur le site forestier de Mbouma-Oyali à la périphérie de Franceville (01'37'29.5', de latitude Sud ; 01341'14.7', longitude Est).

\section{Méthodes}

\section{Mise en place de l'essai}

Les sols témoins provenant de Mbouma-Oyali et les poudres de manganèse ont séparément été séchés pendant 7 jours à l'étuve, à la température fixe de $100{ }^{\circ} \mathrm{C}(\mathrm{Ontod}, 2017)$. Les 2 matériaux séchés ont par la suite été mélangés, de façon homogène, dans des seaux plastiques (contenance $20 \mathrm{~L})$ pour constituer les traitements suivants :

Te : $10 \mathrm{~kg}$ de sol témoin ( $0 \%$ de manganèse) ;

$\mathrm{T} 25: 2,5 \mathrm{~kg}$ de manganèse $+7,5 \mathrm{~kg}$ de sol témoin (25\% de manganèse) ;

T50 : $5 \mathrm{~kg}$ de manganèse $+5 \mathrm{~kg}$ de sol témoin ( $50 \%$ de manganèse) ;

$\mathrm{T} 75: 7,5 \mathrm{~kg}$ de manganèse $+2,5 \mathrm{~kg}$ de sol témoin ( $75 \%$ de manganèse) ; $\mathrm{T} 100$ : $10 \mathrm{~kg}$ de manganèse (100\% de manganèse). 
Pour chaque traitement, 3 seaux ont été constitués. L'échantillon final comptait 15 seaux, soit 45 plants pour les 3 répétitions réalisées. Tous les seaux ont été transférés dans une serre de culture $\left(12 \times 4 \times 3 \mathrm{~m}^{3}\right)$ et ensemencés avec des plants de tubercules par enfouissement à $8 \mathrm{~cm}$ de profondeur. Chaque seau a été arrosé tous les 2 jours avec $100 \mathrm{ml}$ d'eau distillée (Lépengué et al., 2012). Après 90 jours de culture, toutes les plantes ont été déracinées et soumises à différentes analyses biochimiques et physico-chimiques.

\section{Mesure des matières sèches}

Après déracinement, le nombre de tubercules a été compté par seau, et les parties aériennes séparées des racines à l'aide d'un sécateur tranchant (Lépengué et al., 2012). Les différentes parties ont été rincées avec de l'eau de robinet, desséchées entre 2 épaisseurs de papier buvard, enveloppées dans du papier aluminium et pesées (balance Ohaus Analytic 60 ; USA), pour obtenir les masses des matières fraîches des organes. Ensuite, ces organes ont été séchés pendant 15 jours à l'étuve, à la température fixe de $70^{\circ} \mathrm{C}$. Après refroidissement, les échantillons ont à nouveau été pesés pour obtenir les masses des matières sèches des parties aériennes et souterraines (Lépengué et al., 2016).

\section{Dosage des chlorophylles totales}

Les teneurs chlorophylliennes ont été relevées au $90^{\mathrm{e}}$ jour, avant la récolte des plantes, par la méthode non intrusive utilisant un appareil numérique à infra-rouge (Chlorophyll-meter Atleaf+; USA). Les données rapportées sont les moyennes des mesures des 10 premières feuilles apparues sur chaque plante (Mokea-Niaty, 2019).

\section{Dosage des sucres totaux}

Les sucres totaux ont été dosés par la méthode au phénol-sulfurique en utilisant $1 \mathrm{~g}$ de matière sèche de chaque échantillon (Ontod, 2017). Le broyage a été effectué dans $10 \mathrm{ml}$ d'alcool $80^{\circ} \mathrm{GL}$ et centrifugé pendant 10 minutes, à $4000 \mathrm{tr} / \mathrm{min}$. Les impuretés du surnageant ont été précipitées avec $2 \mathrm{ml}$ d'acétate de plomb $10 \%$. L'acétate de plomb excédentaire a été neutralisé, après 10 minutes d'incubation, par ajout de $2 \mathrm{ml} \mathrm{d}$ 'acide oxalique (10\%). L'extrait glucidique a été obtenu après centrifugation de la solution et évaporation du surnageant recueilli sur un bain de sable chauffant pendant 30 minutes. Le dosage a été réalisé à l'aide d'un spectrophotomètre (Spectrophotometer, Unico 1100, UK) à la longueur d'onde de $490 \mathrm{~nm}$ contre un tube témoin. La droite étalon a été tracée à partir d'une solution-mère de glucose concentrée à $1 \mathrm{mg} / \mathrm{ml}$ (Lépengué et al., 2011). 


\section{Analyse statistique}

Les données issues des 3 répétitions ont été soumises à une analyse de variance (ANOVA), au logiciel Statistica 7.2. En cas de différence, les tests de comparaison multiples de Newman-Keuls ont été utilisés au seuil de 5\% (Nzengue et al., 2016).

\section{Résultat}

\section{Effets du manganèse sur la production de matières sèches aériennes par la patate douce}

Les résultats de l'effet des concentrations de manganèse sur l'accumulation des biomasses aériennes des plantes de patate douce sont présentés à la figure 1. L'analyse de cette figure a révélé que les 3 plantes témoins produisaient en moyenne $52 \mathrm{~g}$ de matière sèche aérienne après 90 jours de culture. L'amendement des sols à $25 \%$ de manganèse (T25) a généré une augmentation de synthèse de matière organique jusqu'à $68 \mathrm{~g}$ de matière sèche, à la même période; ce qui équivaut à une induction significative de $30 \%$. Les milieux contenant de fortes teneurs en manganèse (T50, T75 et T100) ont en revanche engendré des pertes de biomasses aériennes des patates douces. Ces baisses respectives de $31 \%, 80,77 \%$ et $100 \%$ ont été statistiquement significatives au seuil de 5\%. Le traitement T100 a, en particulier, totalement inhibé la germination des plantes.

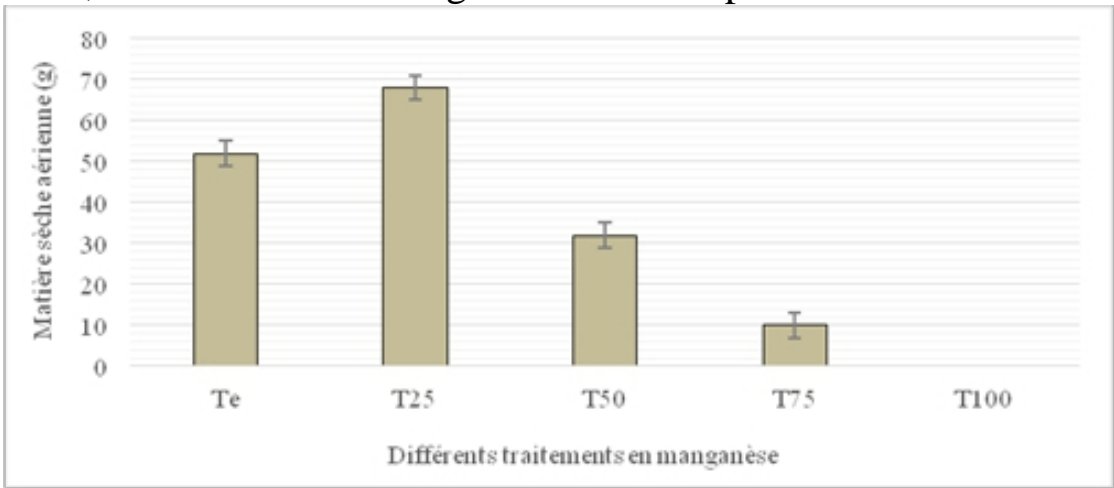

Figure 1 : Production de matière sèche aérienne en fonction des traitements de manganèse.

\section{Effets du manganèse sur la production de biomasse souterraine par la patate douce}

Les résultats de l'effet du manganèse sur la formation de la biomasse souterraine sont assez proches de ceux obtenus précédemment avec la matière sèche aérienne. Les substrats contenant $25 \%$ de manganèse (T25) ont stimulé la formation des organes souterrains (racines et tubercules) des patates douces à hauteur de 22,45\% après 90 jours d'expérimentation. Les 3 autres traitements (T50, T75 et T100) ont en revanche, et comme dans le cas des biomasses aériennes, abaissé la production des matières végétales racinaires. 
Ces pertes significatives de matières souterraines ont respectivement été de $36,76 \%, 77,55 \%$ et $100 \%$.

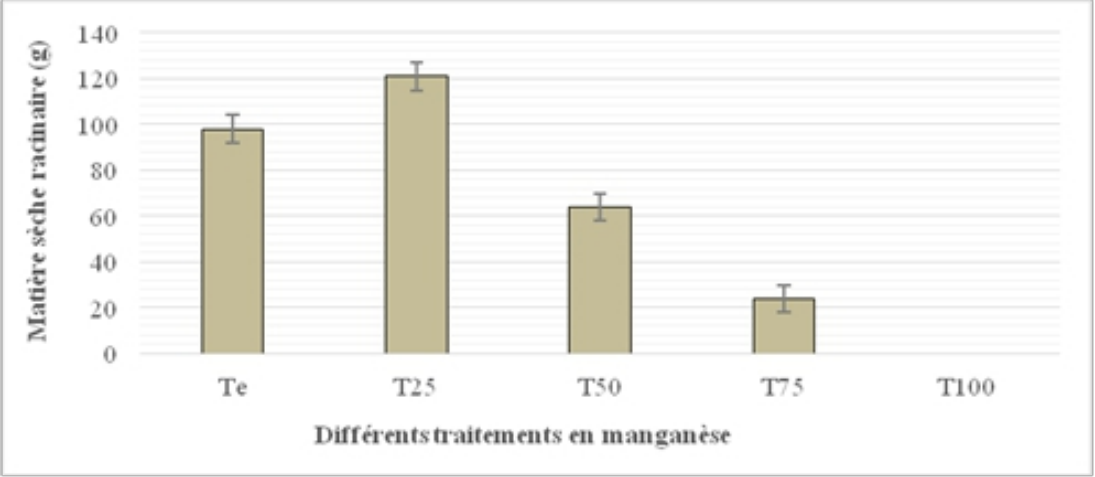

Figure 2 : Production de matière sèche souterraine en fonction des traitements de manganèse.

\section{Effets du manganèse sur la productivité des tubercules par la patate douce}

Après 90 jours de culture, les 3 plantes de patate douce du traitement témoin (Te) ont produit en moyenne 12 tubercules (figure 3). Les amendements en manganèse de $25 \%$ (T25) ont permis d'élever ce nombre à 17 unités. Cette augmentation correspond à une hausse significative de $41,66 \%$. Les forts amendements en manganèse (T50 et T75) ont en revanche significativement ralenti la formation des tubercules pour aboutir aux productions moyennes respectives de 7 et 2 organes à la fin de l'expérimentation. Le traitement T100 n'a produit aucun tubercule de patate douce.

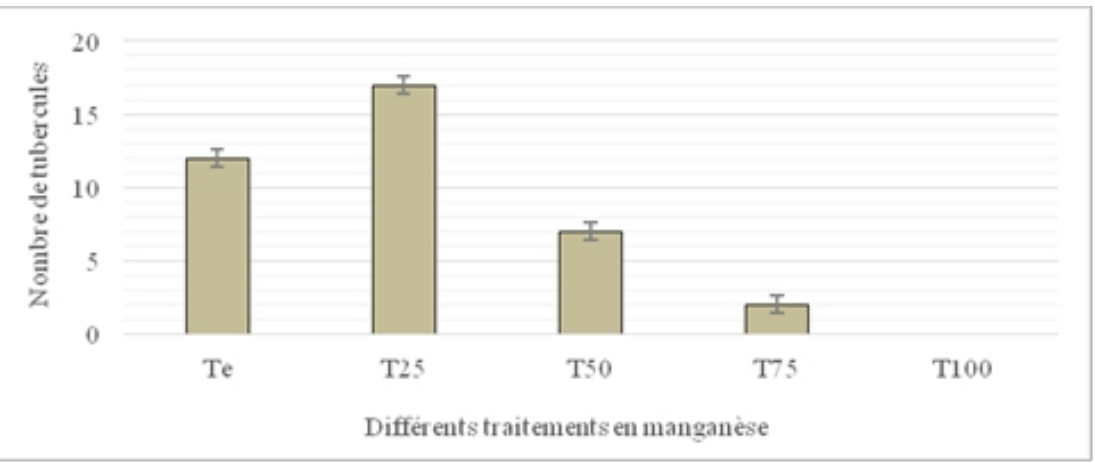

Figure 3: Productivité des tubercules de patate douce en fonction des traitements de manganèse.

Effets du manganèse sur les teneurs chlorophylliennes de la patate douce Les teneurs chlorophylliennes des plantes témoins ont été évaluées à $1,82 \mathrm{mg} / \mathrm{g}$ de matière sèche (MS) (figure 4). Les plantes issues des milieux amendés à $25 \%$ de manganèse (T25) ont présenté des feuilles fortement 
colorées, avec des teneurs chlorophylliennes équivalant à $2,6 \mathrm{mg} / \mathrm{g}$ de MS, à la fin de l'étude au $90^{\mathrm{e}}$ jour. Les traitements T50 et T75 ont engendré des plantes aux feuilles étiolées et déverdies, avec des teneurs chlorophylliennes correspondant respectivement à $1 \mathrm{mg} / \mathrm{g}$ de MS et $0,5 \mathrm{mg} / \mathrm{g}$ de MS. Ces baisses de teneurs chlorophylliennes correspondent à des pertes significatives respectives de $44,44 \%$ et $72,22 \%$, au seuil statistique de $5 \%$.

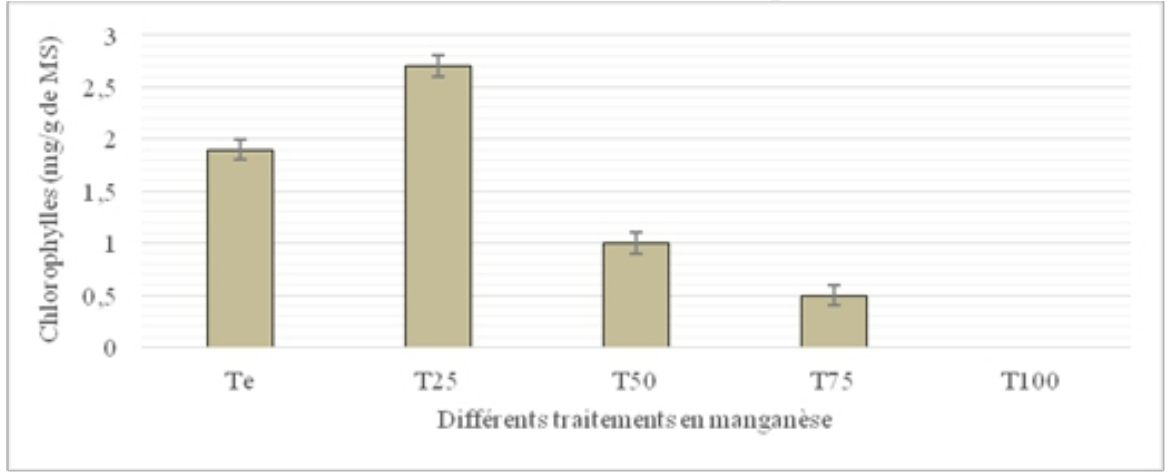

Figure 4 : Teneurs en chlorophylliennes des feuilles en fonction des traitements de manganèse.

\section{Effet du manganèse sur la formation des sucres totaux}

Les résultats de l'effet des différentes teneurs de manganèse sur la formation des sucres totaux dans les tubercules des patates douces sont présentés à la figure 5. Comme pour tous les paramètres morphométriques et biochimiques analysés précédemment, les 2 tendances évolutives ont encore été observées. Les tubercules issus des substrats T25 ont présenté des taux de sucres totaux (64 mg/g de MS) significativement supérieurs à ceux des organes témoins $(10,83 \mathrm{mg} / \mathrm{g}$ de MS). Les organes issus des traitements T50 et T75 ont en revanche présenté des teneurs en sucres plus faibles $(6,93 \mathrm{mg} / \mathrm{g}$ de MS et 3,56 mg/g de MS). Ces teneurs sont significativement inférieures à celles des plantes témoins. Le traitement T100 n'a pas produit de tubercules.

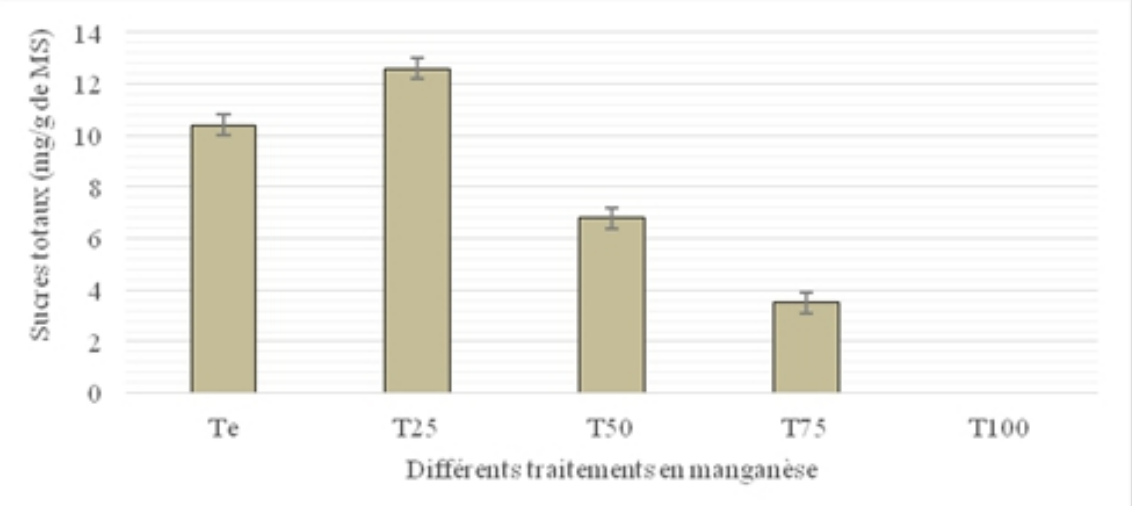

Figure 5 : Teneurs en sucres totaux des tubercules de patate douce en fonction des traitements de manganèse 


\section{Discussion}

Les résultats de la présente étude ont montré que les faibles traitements de manganèse T25 (correspondant à $25 \%$ de manganèse) induisaient l'augmentation des paramètres morphométriques et biochimiques de croissance des patates douces. En effet, tous les 3 paramètres morphométriques analysés (matière sèche aérienne, matière sèche racinaire et nombre de racines) ont présenté pour ce traitement des hausses de valeur supérieures à 20\%, comparativement au témoin. La hausse des paramètres biochimiques a, quant à elle, été dans les deux cas supérieure à 30\%.

Les traitements de plus grandes concentrations en manganèse (T50 et T75) ont en revanche retardé la croissance des paramètres morphométriques et biochimiques de plus de 40\%. Le traitement T100 (correspondant à 100\% de manganèse) a totalement inhibé la croissance des plants de patate douce.

Tous ces résultats antagonistes en fonction de la concentration en $\mathrm{Mn}$, c'est-à-dire la stimulation et le ralentissement des paramètres de croissance végétale sont en accord avec ceux de Mombo et al. (2017) et Nzengue et al. (2019) sur le manioc (Manihot esculenta Crantz) et l'arachide (Arachis hypogeae L.). En effet, les travaux de ces auteurs ont révélé une induction de la croissance longitudinale et radiale des tiges soumises à des traitements manganifères de $25 \%$. Des résultats similaires ont été signalés par Ontod et al. (2013) sur les plantes de roselle (Hibiscus sabdariffa L.var. sabdariffa).

La stimulation de la croissance végétale par de petites concentrations de manganèse semble liée au rôle majeur joué par cet oligoélément dans la phase lumineuse de la photosynthèse (Heller et al., 2002). En effet, la photosynthèse se compose de 2 phases couplées, l'une dite lumineuse (réactions photochimiques), et l'autre sombre ou obscure (réactions chimiques). Le manganèse est un intermédiaire de la phase lumineuse (schéma en $\mathrm{Z}$ ) accepteur d'électrons provenant de la photolyse de l'eau, situé au potentiel $+0,82 \mathrm{~V}$, entre l'eau et le photosystème II (Heller et al., 2002). L'addition de manganèse à faible concentration dans le milieu de culture de la patate douce augmenterait le potentiel de réception des électrons, donc une activation de la production d'énergie au cours de cette phase. La fixation de $\mathrm{CO}_{2}$ s'en trouverait également accélérée et aboutirait à une hausse de production des sucres, donc de la matière organique (Hopkins, 2003). C'est cette plus-value énergétique qui aboutirait à la hausse des paramètres de croissance observée chez la patate douce. Des résultats similaires ont également rapportés par Lépengué et al. (2012) sur le maïs, Mavoungou et al. (2016) sur la roselle et Ontod et al. (2015) sur le manioc.

La réduction des paramètres morphométriques et biochimiques (ou leur inhibition) observée au niveau des teneurs élevées de manganèse (T50, T75 et T100) pourrait être due à des phénomènes de toxicité chimique engendrés par l'excès de Mn. En effet, les travaux de Ontod et al. (2013) ont rapporté le 
potentiel toxique du manganèse à des teneurs élevées supérieures à 50\%, sur les plantes de roselle, au Gabon. Il s'agirait d'un déficit de sites de fixation de cet oligoélément sur les organites cellulaires des plantes hôtes (Hopkins, 2003). Les travaux de Mombo et al. (2017) ont également révélé une perturbation de la nutrition minérale des plantes de manioc cultivées sur des milieux de concentrations élevées en manganèse à Moanda, au Gabon. Cette perturbation était caractérisée par une réduction de l'absorption des cations $\mathrm{Ca}^{2+}$ et une augmentation de celle des $\mathrm{K}^{+}$. La rupture de l'équilibre d'absorption pourrait expliquer la carence du calcium et à l'excès du potassium aboutissant à la réduction des paramètres morphologiques et biochimiques des plantes. Toutes ces perturbations pourraient survenir au cours de la croissance de la patate douce, et aboutir à la baisse des paramètres de croissance notée chez les plantes cultivées sur des sols fortement enrichis en manganèse.

\section{Conclusion}

Les différents traitements de manganèse sur les patates douces en serre ont montré que les teneurs minérales de $25 \%$ induisaient la hausse des paramètres morphométriques (matières sèches aériennes et souterraines et nombre de tubercules) et biochimiques (teneurs en chlorophylles et en sucres totaux) étudiés. Les traitements manganifères de concentrations élevées $(50 \%$, $75 \%$ et $100 \%$ ) ont tous réduit les teneurs des paramètres précités. Les effets du traitement T100 (ne contenant que du manganèse) ont été tellement sévères, qu'aucune plante n'a poussé dans ce milieu. La patate douce est donc cultivable dans la zone de Moanda, mais uniquement sur des sites de faible concentration manganifère $(25 \%$ de $\mathrm{Mn})$. Dans ces milieux, la concentration en Mn des sols stimule la croissance et le développement de la patate douce. Néanmoins, des études complémentaires sur les mesures de bioaccumulation du Mn dans les différentes parties comestibles de la patate douce (feuilles et tubercule) doivent être réalisées pour limiter les risques d'exposition des personnes qui les consomment quotidiennement dans cette région du Gabon.

\section{Remerciements}

Nous exprimons nos remerciements à la Compagnie Minière de l'Ogooué (COMILOG) pour les terrils de manganèse mis à notre disposition.

\section{References :}

1. Aterianus-owanga, A. et Debain, M. Un renversement électoral confisqué au Gabon. Politique Africaine in Cairn. Info Pour Editions Karthala, 144 : 157-179; 2016.

2. Boupassia, C. Étude des sols et des résidus miniers de la région de Moanda au Sud-Est du Gabon: Perspectives de réhabilitation du 
plateau manganésifère de Bangombé. Thèse de doctorat en Sciences de la Terre et de l'Environnement. Université de Bourgogne (France) 305 p., 2004.

3. Clarke, C. and Upson, S. A global portrait of the manganese industryA socioeconomic perspective. Neuro Toxicology, 58 : 173-179; 2017.

4. COMILOG. Le premier pas. Rapport annuel, 68 p., 2018.

5. Eba, F., Ondo, J.A., Emane, M.S., Ollui-M'boulou, M. and Omva-Zue, J. Taux de manganèse accumulé dans quelques plantes vivrières cultivées dans la région manganésifère de Moanda (Gabon). J. Soc. Ouest-Afr. Chim., $023: 69-74 ; 2007$.

6. Goix, S., Mombo, S., Schreck, E., Pierart, A., Lévêque, T., Deola, F. and Dumat, C. Field isotopic study of lead fate and compartmentalization in earthworm-soil-metal particle systems for highly polluted soil near $\mathrm{Pb}$ recycling factory. Chemosphere, 138 : 10$17 ; 2015$.

7. Heller, R., Esnault, R. and Lance, C. Physiologie végétaleDéveloppement. 6ième édition, Éditeur Dunod, collection Sciences sup., Paris, 384 p., 2002.

8. Hopkins, W.G., Physiologie végétale. Edition de Boeck, Université de Bruxelles, Belgique, 532 p., 2003.

9. Lebas, L. Impacts de l'exploitation minière sur les populations locales et l'environnement dans le Haut-Ogooué. Rapport d'Etude de l'ONG Brainforest, 47 p., 2010.

10. Lépengué, A.N., Mouaragadja, I., Cherif, M., Aké, S. et M'batchi, B. Amélioration de la croissance du maïs (Zea mays L. var. LG 60, Poaceae) par des traitements à la colchicine. Journal of Applied Biosciences, 52 : 3660-3668; 2012.

11. Lépengué, A.N., Souza, A., Yala, J.F., Lebamba, J., Mavoungou, J.F. et M'batchi, B. Etude de quelques caractéristiques physicochimiques et biochimiques de Wavé-fortex un complément alimentaire naturel du Gabon.European Scientific Journal, 12 (33) : 508-520 ; 2016.

12. Lépengué, A.N., Yala, J.F., Mouaragadja, I., Ontod, T.D.S., Mbadoumou, NB., Mokéa, N.A., Aké, S. et M'batchi, B. Rôle de l'acide borique dans la synthèse de quelques composés biochimiques de la roselle (Hibiscus sabdariffa L.var. sabdariffa) au Gabon. Revue du CAMES, Sciences et Médecine, 12 (2) : 216-220 ; 2011.

13. Lévêque, T., Capowiez, Y., Schreck, E., Mombo, S., Mazzia, C., Foucault, Y. and Dumat, C. Effects of historic metal (loid) pollution on earthworm communities. Science of The Total Environment, 511: $738-746 ; 2015$.

14. Leveque, T., Capowiez, Y., Schreck, E., Xiong, T., Foucault, Y. and Dumat, C. Earthworm bioturbation influences the phytoavailability of 
metals released by particles in cultivated soils. Environmental pollution (Barking, Essex : 1987), 191C : 199-206 ; 2014.

15. Mavoungou, A.Y., Lepengué A.N. and M'batchi, B. Antioxydants et phytochelatines dans la tolérance et l'accumulation du manganèse chez Hibiscus sabdariffa Linn. European Scientific Journal, 11 (21) : 430444 ; 2016.

16. Mavoungou, A.Y., Lepengué A.N. and M'batchi, B. Manganese's effect in the sheet apoplast Hibiscus sabdariffa L. var sabdariffa for 10 days. European Scientific Journal, 11 (24) : 73-47 ; 2015.

17. Messi-Me, N.A.N. Impact des éléments traces métalliques sur les plantes alimentaires de la ville minière de Moanda (Gabon). Cas du Manihot esculenta Crantz. These de doctorat en chimie inorganique, specialité: Chimie de l'environnement. Université de Sciences et Techniques de Masuku, 132 p., 2018.

18. Mokea-Niaty, A. Alchornea cordifolia (Schumach. \& Thonn.) Müll. Arg., Euphorbiaceae : candidate potentielle à la phytoremédiation des sols pollués en manganèse. Thèse de doctorat en chimie inorganique, spécialité: Biotechnologie et Ecophysiologie végétales. Université de Sciences et Techniques de Masuku, 294 p., 2019

19. Mombo, S., Foucault, Y., Deola, F., Gaillard, I., Goix, S., Shahid, M., Schreck, E., Pierart, A. and Dumat, C. Management of human health risk in the context of kitchen gardens polluted by lead and cadmium near a lead recycling company. Journal of Soils and Sediments, 16 (4): $1214-1224 ; 2015$.

20. Mombo, S., Laplanche, C., Besson, P., Sammartino, S., Schreck, E., Dumat, C. and Capowiez, Y. Metal soil pollution differentially affects both the behaviour and exposure of A. caliginosa and L. terrestris: a mesocosm study. Biology and Fertility of Soils, 54 (3): 319-328 ; 2018.

21. Mombo, S., Lepengué, A.N., Muloway, K.A., Souza, A., Mbatchi, B., Ontod, T.D.S. et Dumat, C. Etude de l'impact du manganèse sur l'absorption minérale du manioc (Manihot esculenta Crantz) dans les sols manganifères au Gabon et de ses potentielles conséquences environnementales et sanitaires. Sustainable Urban Agriculture (Open Archive Toulouse), 1 p., 2017.

22. Ndala, P. D. I. et Moussone, E. Les opportunités des investissements directs étrangers au Gabon. L'Harmattan «Marché et organisations » in Cairn. Info Pour Editions Karthala, 2 (14) : 57-95 ; 2011.

23. Nguéma, N. S., Kombila, C. G., Bibang, J. N., Tongo, P., Zue, E. P. Etat des lieux environnemental: Rivière la Moulili aval de Moanda. Rapport d'Etude de l'ONG Brainforest 54 p., 2014. 
24. Nzengue, E., Iponga, M. D., M'Sadak, Y., Owona, A. G. S., ZingaKoumba C. R., Assani, S., M'batchi B., Mavoungou J. F., Production des plants de Tomate (Lycopersicon esculentum) sur substrats de culture à base de parche de café à différents gradients de désinfection dans une pépinière maraîchère hors sol au Gabon. International Journal of Innovation and Scientific Research, 26 (1) : 83-94 ; 2016.

25. Nzengue, E., Lepengué, A.N., Mbeang B. A. M.,Mombo, S.,Ontod, T.D.S., Mokéa, A., Mavoungou, J. P., Ngomanda A., Midoko, I.D., M'batchi,B. Effets des terrils de manganèse de la mine de Moanda (Gabon) sur quelques caractères morphométriques de l'Arachide (Arachis hypogea L.). European Journal Scientific Research, 153 (3) : 335-352; 2019.

26. Ontod, T.D.S. Effets de la pollution manganifère, sur la croissance et le rendement du manioc (Manihot esculenta CRANTZ) cultivé en serre dans le Sud Est du Gabon. Thèse de doctorat de l'Université de Sciences et Techniques de Masuku (USTM)-Gabon. Ecole doctorale en Sciences Fondamentales et appliquées, option BiologiePhytoremédiation. 210 p., 2017.

27. Ontod, T.D.S., Lepengué, A.N. et M'batchi, B. Effet de la toxicité manganifère sur les paramètres morphométriques de la roselle (Hibiscus sabdariffa L.var.sabdariffa) au Gabon. European Scientific Journal, 9 (15) : 203-218; 2013.

28. Ontod, T.D.S., Lepengue, A.N., Mbatchi, B., Effet Des Concentrations En Manganèse du sol sur la croissance du Manioc (Manihot Esculenta Crantz) Au Gabon. European Scientific Journal, 11 (33) : 281-295 ; 2015.

29. Xiong, T., Leveque, T., Shahid, M., Foucault, Y., Mombo, S. and Dumat, C. Lead and Cadmium Phytoavailability and Human Bioaccessibility for Vegetables Exposed to Soil or Atmospheric Pollution by Process Ultrafine Particles. Journal of Environment Quality, 43 (5) : 1593-6000; 2014. 